\title{
ON RELATIVELY NONATOMIC MEASURES
}

\section{J. R. BLUM}

1. Introduction and summary. Let $\Omega$ be a set, $a$ a $\sigma$-algebra of subsets of $\Omega$, and $m$ be a measure defined on $Q$ whose range $R$ is a subset of $k$-dimensional Euclidean space $E_{k}$, i.e., $m$ is a completely additive set function defined on $a$ such that each component of $m$ is nonnegative. If each component of $m$ is nonatomic it was shown by Lyapunov [3], Halmos [2], and Blackwell [1] that $R$ is a convex set. Now let $a$ be the class of Borel subsets of the real line, let $m=\left(m_{1}, m_{2}\right)$ where $m_{1}$ is Lebesgue measure and $m_{2}(A)$ counts the number of integers in the set $A$. Then $R$ is not convex, in fact $R$ is the set of points $\{(x, y)\}$ where $x \geqq 0$ and $y$ is a nonnegative integer. Yet if $(x, y) \in R$ and $(x, y)$ lies on the line segment connecting the zero vector and $m(A)$ it is easily seen that there exists $A^{\prime} \subset A$ such that $m\left(A^{\prime}\right)=(x, y)$. In this note we give a definition of relative nonatomicity which covers situations of this kind and prove the analogue of the convexity theorem.

2. Definitions and results. We shall assume throughout that every subset of $\Omega$ that is discussed is measurable, i.e., an element of $a$.

Definition 1. Let $R$ be the range of $m . m$ is nonatomic relative to $R$ if for every set $A$ and every number $\alpha$ with $0<\alpha<1$ such that $\alpha m(A)$ $\in R$ there exists $A^{\prime} \subset A$ and a number $\alpha^{\prime}$ with $0<\alpha^{\prime}<1$ such that $m\left(A^{\prime}\right)=\alpha^{\prime} m(A)$.

Definition 2. Let $A$ be a set. We define $R(A)=\{r \in R \mid r=\alpha m(A)$ with $0<\alpha \leqq 1\}$. Let $\alpha_{0}=\inf \{\alpha \mid \alpha>0, \alpha m(A) \in R\}$. Then we define $r_{0}(A)=\alpha_{0} m(A)$.

Theorem. Let $m$ be nonatomic relative to $R$. Let $A$ be a set and $r \in R(A)$. Then there exists $B \subset A$ with $r=m(B)$.

The proof of the theorem will proceed by way of several lemmas.

Lemma 1. In Definition 1 we may choose $A^{\prime}$ and $\alpha^{\prime}$ such that $\alpha^{\prime} \leqq \alpha$.

Proof. Suppose the conclusion of the lemma is false. Let $A^{\prime} \subset A$ such that $m\left(A^{\prime}\right)=\alpha^{\prime} m(A)$ with $0<\alpha^{\prime}<1$. Then $m\left(A-A^{\prime}\right)$ $=\left(1-\alpha^{\prime}\right) m(A)$ and $\alpha^{\prime}>\alpha, 1-\alpha^{\prime}>\alpha$. Hence $\alpha<1 / 2$. Now $r=\alpha m(A)$ $=\left(\alpha / \alpha^{\prime}\right) \alpha^{\prime} m(A)=\left(\alpha / \alpha^{\prime}\right) m\left(A^{\prime}\right)$, and similarly

$$
r=\left[\alpha /\left(1-\alpha^{\prime}\right)\right] m\left(A-A^{\prime}\right) .
$$

Hence we may apply the definition and the above procedure separately to $A^{\prime}$ and $A-A^{\prime}$. After some manipulation we obtain $\alpha<1 / 4$.

Received by the editors March 2, 1960 and, in revised form, May 27, 1960. 
Repeating this process indefinitely yields $\alpha=0$, which is a contradiction.

Lemma 2. Suppose $m$ is nonatomic relative to $R$. Then for every set $A$ there exists $B \subset A$ such that $m(B)=r_{0}(A)=\alpha m(A)$ for some $\alpha$, with $0 \leqq \alpha \leqq 1$.

Proof. If $r_{0}(A)$ is the null vector choose $B$ to be the empty set and $\alpha=0$, and if $r_{0}(A)=m(A)$ choose $B=A$ and $\alpha=1$. If $r_{0}(A) \in R(A)$ the conclusion follows from Lemma 1 . Otherwise there exists a strictly decreasing sequence of numbers $\left\{\alpha_{n}\right\}$ with $0<\alpha_{n}<1$ such that $r_{0}(A)$ $=\lim _{n} \alpha_{n} m(A)$, and such that $\alpha_{n} m(A) \in R(A)$. But then it follows again from Lemma 1 that there exists a sequence of number $\left\{\alpha_{n}^{\prime}\right\}$ and a sequence of sets $\left\{B_{n}\right\}$ which may be chosen so that $A \supset B_{1}$ $\supset B_{2} \supset \ldots$ such that $\lim _{n} m\left(B_{n}\right)=\lim _{n} \alpha_{n}^{\prime} m(A)=r_{0}(A)$. It $B=\lim _{n} B_{n}$ then clearly $B$ satisfies the conclusion of the lemma.

Lemma 3. Suppose $m$ is nonatomic relative to $R$. Let $A$ be a set and suppose $B \subset A$ satisfies the conclusion of Lemma 2, i.e., $m(B)=r_{0}(A)$. Then either $m(B)$ is the null vector or $m(A)$ is an integral multiple of $m(B)$.

Proof. Suppose $m(B)$ is not the null vector, i.e., $m(B)=\alpha m(A)$ for some $\alpha$ with $0<\alpha \leqq 1$. If $\alpha=1$ we are done. Assume then that $0<\alpha<1$. Choose $B^{\prime} C A-B$ so as to satisfy Lemma 2, i.e., $m\left(B^{\prime}\right)=r_{0}(A-B)$ $=\alpha^{\prime} m(A-B)=\alpha^{\prime}(1-\alpha) m(A)$ for some $\alpha^{\prime}$ with $0 \leqq \alpha^{\prime} \leqq 1$. From the definition of the function $r_{0}$ it follows that $\alpha^{\prime}(1-\alpha) \geqq \alpha$. If $\alpha<\alpha^{\prime}(1-\alpha)$ we may write $m(B)=\left[\alpha /\left(\alpha^{\prime}(1-\alpha)\right)\right] m\left(B^{\prime}\right)$. It then follows from the definition of relative nonatomicity that there exist $B^{\prime \prime} C B^{\prime}$ and $\alpha^{\prime \prime}$ with $0<\alpha^{\prime \prime}<1$ such that $m\left(B^{\prime \prime}\right)=\alpha^{\prime \prime} m\left(B^{\prime}\right)$. But this contradicts the fact that $B^{\prime}$ is minimal for $A-B$, i.e., $m\left(B^{\prime}\right)=r_{0}(A-B)$. Hence $m\left(B^{\prime}\right)=m(B)$. Now if $m\left(A-B-B^{\prime}\right)$ is the null vector we are done. Otherwise we repeat this process. But clearly this must stop in a finite number of steps, and the lemma is proved. By the same techniques we have immediately

Lemma 4. Suppose $m$ is nonatomic relative to $R$. Let $A$ be a set and suppose $r_{0}(A)$ is not the null vector. Then $A$ is the union of finitely many disjoint sets, each having measure $r_{0}(A)$.

Lemma 5. Suppose $m$ is nonatomic relative to $R$. Let $A$ be a set and suppose $r_{0}(A)$ is not the null vector. Then every $r \in R(A)$ is a positive integral multiple of $r_{0}(A)$.

Proof. Let $r \in R(A)$, i.e., $r=\alpha m(A)$ with $0<\alpha \leqq 1$. From Lemma 3 
it follows that there exists $B C A$ such that $m(A)=n m(B)=n r_{0}(A)$ for some positive integer $n$. If $\alpha=1$ there is nothing to prove. Assume then that $0<\alpha<1$ and that $\alpha=(k+c) / n$ where $k$ is an integer with $1 \leqq k<n$ and $c$ is a number with $0<c<1$. The case $k=0$ is impossible for in that case $\alpha<1 / n$. Now $r \in R$ and hence there exists a set $C$ such that $r=m(C)$. Now consider $r_{0}(C)=\alpha^{\prime} m(C)=\alpha^{\prime} \alpha m(A)$ for some $\alpha^{\prime}$ with $0 \leqq \alpha^{\prime} \leqq 1$. If $\alpha^{\prime}=0$ then $r_{0}(A)$ is the null vector which is contrary to the hypothesis. Consequently $\alpha^{\prime}>0$ and $m(C)=i m\left(C_{1}\right)$ for some set $C_{1} \subset C$ and some positive integer $i$, from Lemma 3. Now $m(B)=(1 / n) m(A)=(1 /(k+c)) m(C)$ and hence $(1 / i) \leqq(1 /(k+c))$. Since $k+c$ is not an integer we have $k+c<i$. But then $m\left(C_{1}\right)$ $=[(k+c) /(i n)] m(A)$ and $(k+c) /(i n)<1 / n$ which contradicts the minimality of $1 / n$. Hence $k+c$ must be an integer and the lemma is proved.

Proof of the theorem. If $r_{0}(A)$ is not the null vector the conclusion of the theorem follows at once from Lemma 4 and Lemma 5. Suppose then that $r_{0}(A)$ is the null vector. Now $r=\alpha m(A)$ for some $\alpha$ with $0 \leqq \alpha \leqq 1$. If $\alpha=0$ or $\alpha=1$ the conclusion is trivial. Assume then that $0<\alpha<1$. Let $\mathcal{F}=\{B \subset A \mid m(B)=\beta m(A)$ with $0<\beta \leqq \alpha\}$. We partially order $\mathcal{F}$ by saying that $B_{1}<B_{2}$ if $B_{1} \subset B_{2}$ and if the corresponding $\beta_{1}$ and $\beta_{2}$ satisfy $\beta_{1}<\beta_{2}$. If $\mathcal{F}^{\prime}$ is a linearly ordered subfamily of $\mathcal{F}$ it is easily seen that $\mathcal{F}^{\prime}$ has an upper bound in $\mathcal{F}$. Consequently Zorn's lemma applies. Let $B$ be a maximal element of $\mathcal{F}$ and suppose $m(B)=\beta m(A)$. We shall show that $\beta=\alpha$. Suppose $\beta<\alpha$. Since $r_{0}(A)$ is the null vector it follows easily that $r_{0}(A-B)$ is the null vector. Hence we can find an arbitrarily small positive number $\gamma$ and a corresponding set $B^{\prime} \subset A-B$ such that $m\left(B^{\prime}\right)=\gamma m(A-B)$ $=\gamma(1-\beta) m(A)$. Let $B^{\prime \prime}=B \cup B^{\prime}$. Then $m\left(B^{\prime \prime}\right)=[\beta+\gamma(1-\beta)] m(A)$ and by choosing $\gamma$ sufficiently small we violate the maximality of $B$. Thus $\beta=\alpha$ and theorem is proved.

\section{REFERENCES}

1. D. Blackwell, The range of certain vector integrals, Proc. Amer. Math. Soc. vol. 2 (1951) pp. $390-395$.

2. P. Halmos, The range of a vector measure, Bull. Amer. Math. Soc. vol. 54 (1948) pp. 416-421.

3. A. Lyapunov, Sur les fonctions-vecteurs complètement additives, Bull. Acad. Sci. URSS. Sér. Math. [Izvestia Akad. Nauk SSSR.] vol. 4 (1940) pp. 465-478.

SANDia Corporation 